CASE REPORT

\title{
Intravascular ultrasound findings of coronary wall morphology in a patient with pseudoxanthoma elasticum
}

\author{
K Miwa, T Higashikata, H Mabuchi
}

Heart 2004;90:e61 (http://www.heartinl.com/cgi/content/full/90/10/e61). doi: 10.1136/hrt.2004.040592

Pseudoxanthoma elasticum (PXE) is an inherited disorder characterised by progressive calcification of the elastic fibres in the skin, eye, and cardiovascular system. Recently, mutations in the ATP binding cassette transporter gene (ABCC6) were identified as cause of this disease. Although patients with PXE often have coronary artery disease, little is known about the process and the mechanism of coronary artery disease in PXE. In this report, intravascular ultrasound (IVUS) imaging was performed in a female patient with PXE seven years after the onset of skin lesion to assess the coronary wall morphology in detail. IVUS showed a unique five layer appearance without acoustic shadowing along the vessel wall observed in the angiographically normal portion. These findings may reflect the earlier stage of coronary artery disease caused by PXE before calcification of the internal elastic laminae.

$\mathrm{P}$ seudoxanthoma elasticum (PXE) is an inherited disorder characterised by progressive calcification of the elastic fibres in the skin, eye, and cardiovascular system. ${ }^{1-3}$ The prevalence of PXE in the general population is unknown, particularly because patients with a mild clinical phenotype often escape diagnosis. Recently mutations in the ATP binding cassette transporter gene (ABCC6) were identified as a cause of this disease. ${ }^{4-6}$ However, little is known about how the coronary artery lesions of PXE associated calcification develop in the internal elastic laminae. Coronary angiography is the standard method to evaluate coronary artery lesions, although the assessment of wall morphology is difficult. Recent progress in intravascular ultrasound (IVUS) technology allows detailed visualisation of the morphological

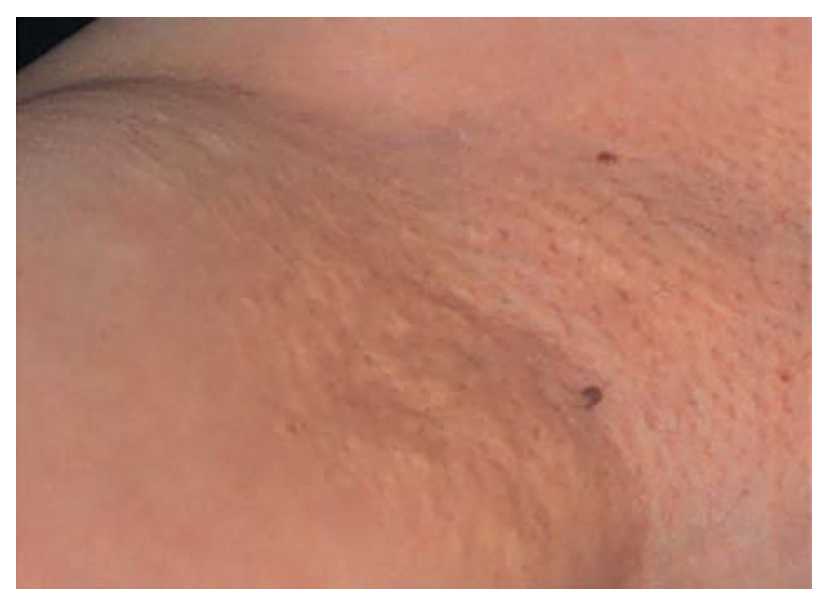

Figure 1 Typical yellowish papules on the right axilla.

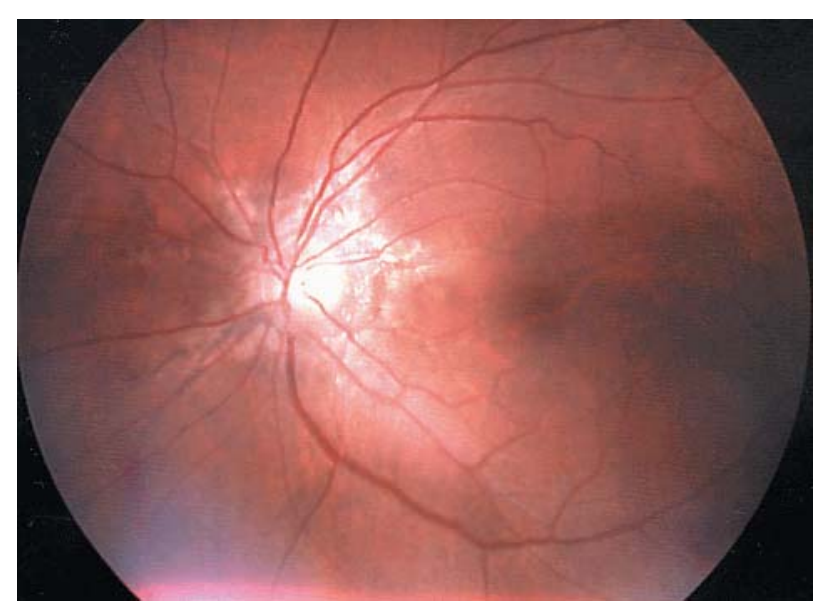

Figure 2 Ophthalmological examination shows the presence of angioid streaks in the left optic fundus.

changes in the vascular wall. This is the first case report of IVUS findings in the coronary artery of a patient with PXE.

\section{CASE REPORT}

A 52 year old Japanese woman was admitted to our hospital for clinical evaluation for PXE in March 2002. She had noticed yellowish papules on her bilateral axillae (fig 1) since she was 45 years old. At age 51, she began to have mild visual impairment and hence visited our hospital. Ophthalmological examination showed angioid streaks in the bilateral retina (fig 2), which is a major clinical feature of PXE. A skin biopsy from the axilla showed characteristic clumping of elastic tissue in the deep dermis and confirmed the diagnosis of PXE. This patient had hypertension, a 20 year history of smoking, and no other conventional risk factors for coronary artery disease.

Chest radiography showed mild calcification of the descending thoracic aorta. ECG and echocardiographic findings were normal. Blood cell counts, blood chemistry tests, and urinalysis were within normal limits. Selective coronary angiography showed no stenotic lesion except for a slightly irregular surface on the proximal portion of the left anterior descending (LAD) and right coronary arteries. IVUS was performed to obtain more information about the vascular wall changes. The Insight and Clear View Systems (cardiovascular imaging systems, CIVIS) were used in this study with imaging catheter $(40 \mathrm{MHz}$ Atlantis/Boston Scientific, Natick, Massachusetts, USA). Through a 6 French guiding catheter, an IVUS transducer was introduced into the middle portion of the LAD. Real time cross sectional images of the LAD were obtained on ECG gated autopullback

Abbreviations: IVUS, intravascular ultrasound; LAD, left anterior descending coronary artery; PXE, pseudoxanthoma elasticum 

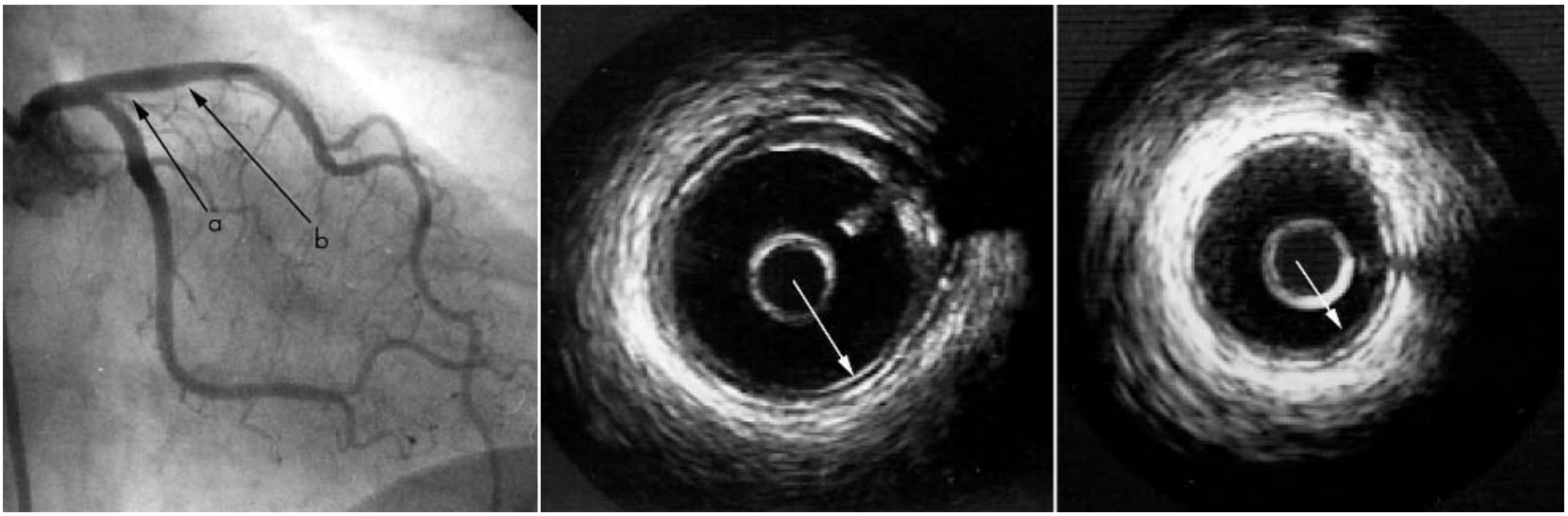

Figure 3 Coronary angiogram and intravascular ultrasound (IVUS) images from the left anterior descending coronary artery (LAD). The IVUS image from the proximal LAD (middle; $a$ in the angiogram) shows mild atherosclerotic plaque (mixed plaque) with a unique five layer appearance of the arterial wall. The IVUS images adjacent to the plaque lesion (angiographically normal portion) (right; $b$ in the angiogram) also show a five layer appearance of the arterial wall. These five layer portions are not accompanied by acoustic shadowing (arrows).

at a speed of $0.3 \mathrm{~mm} / \mathrm{beat}$ (TomTec IVUS scan system, Hamden, Connecticut, USA) and images were continuously recorded on videotape (S-VHS). The proximal portion of LAD, an angiographically irregular site, had mild atherosclerotic plaque with partial acoustic shadowing. The vessel wall had a unique five layer appearance, in contrast to the three layer appearance of a normal vessel wall, with a bright echo signal inserted between the internal and external elastic lamina (fig 3). Interestingly this five layers portion was also present in the angiographically completely normal portion.

\section{DISCUSSION}

This is the first case report of a patient with PXE who was evaluated with IVUS imaging of the coronary artery. In two large clinical series, $13 \%$ and $52 \%$ of patients with PXE had angina pectoris ${ }^{7}$; in some extreme cases, angina pectoris and myocardial infarction occurred before the age of 30 . According to necropsy reports, 14 of $18(78 \%)$ had some degree of coronary atherosclerosis or calcification. ${ }^{9}$

Changes in the vascular wall structure in PXE appear as calcification of the elastic layer of the media in small and medium sized arteries, which is followed by calcification of the intima and the development of atheromatous plaques. ${ }^{7}$

A normal coronary artery wall has three layers on an IVUS image: high, low, and high echo signals, which are equivalent to endothelium and internal elastic lamina, medial layer, and external elastic lamina, respectively. In contrast, the IVUS image of the proximal LAD in this case was shown to have five layers, with an additional bright echo signal inserted between the internal and external elastic laminae. It must be emphasised that this finding was from an angiographically normal portion and was not accompanied by acoustic shadowing. This subclinical pathological change was thought to be different from the characteristic features of PXE such as calcification of the internal elastic lamina and stenotic lesions. Mendelsohn and colleagues ${ }^{1}$ reported from three necropsy cases of PXE that the earliest change histologically detectable in peripheral muscular arteries was fragmentation of the internal elastic lamina and occasionally of the external elastic lamina. Thus, development of the additional bright echo signal may reflect an earlier stage of coronary artery lesion development.

In summary, we report the first IVUS findings in the coronary artery in a patient with PXE, which showed characteristic findings suggesting a preclinical, early stage of coronary artery involvement in PXE. Many more cases will need to be examined to elucidate the mechanism of onset and development of coronary artery involvement in PXE.

\section{Authors' affiliations}

K Miwa, T Higashikata, H Mabuchi, Molecular Genetics of Cardiovascular Disorders, Division of Cardiovascular Medicine, Graduate School of Medical Science, Kanazawa University, Kanazawa, Japan

Correspondence to: Dr Kenji Miwa, Molecular Genetics of Cardiovascular Disorders, Division of Cardiovascular Medicine, Graduate school of Medical Science, Kanazawa University, Takara-machi 13-1, Kanazawa 920-8641, Japan; miwakc2004@ yahoo.co.jp

\section{Accepted 24 May 2004}

\section{REFERENCES}

1 Mendelsohn G, Bulkley BH, Hutchins GM. Cardiovascular manifestations of pseudoxanthoma elasticum. Arch Pathol Lab Med 1978;102:298-302.

2 Lebwohl M, Schwartz E, Lemlich G, et al. Abnormalities of connective tissue components in lesional and non-lesional tissue of patients with pseudoxanthoma elasticum. Arch Dermatol Res 1993;285:121-6.

3 Hausser I, Anton-Lamprecht I. Early preclinical diagnosis of dominant pseudoxanthoma elasticum by specific ultrastructural changes of dermal elastic and collagen tissue in a family at risk. Hum Genet 1991;87:693-700.

4 Bergen AA, Plomp AS, Schuurman EJ, et al. Mutations in ABCC6 cause pseudoxanthoma elasticum. Nat Genet 2000;25:228-31.

5 Germain DP, Perdu J, Remones V, et al. Homozygosity for the R1268Q mutation in MRP6, the pseudoxanthoma elasticum gene, is not diseasecausing. Biochem Biophys Res Commun 2000;274:297-301.

6 Le Saux O, Urban Z, Tscuch C, et al. Mutations in a gene encoding an ABC transporter cause pseudoxanthoma elasticum. Nat Genet 2000;25:223-7.

7 Neldner KH. Pseudoxanthoma elasticum. Clin Dermatol 1988;6:1-159.

8 Carlborg U, Ejrup B, Gronblad E, et al. Vascular studies in pseudoxanthoma elasticum and angioid streaks. Acta Med Scand 1959;166(suppl 350):1-84.

9 Notle KB. Sudden cardiac death owing to pseudoxanthoma elasticum: a case report. Hum Pathol 2000;31:1002-4. 